\title{
Augmented ARCH Models for Financial Time Series: Stability conditions and empirical evidence
}

\author{
Robert M. Kunst \\ Johannes Kepler University \\ A-4040 Linz-Auhof, Austria \\ and \\ Institute for Advanced Studies \\ Stumpergasse 56, A-1060 Vienna, Austria \\ Applied Financial Economics, 1997, 7, 575-586
}

\begin{abstract}
The class of conditionally heteroskedastic models known as 'augmented ARCH' encompasses most linear 'ARCH'-type models found in the literature and, in particular, two basic $\mathrm{ARCH}$ variants for autocorrelated series: Engle (1982) explains conditional variance by lagged errors, Weiss (1984) also by lagged observations. The framework permits an evaluation of whether the restrictions evolving from the Engle or the Weiss models are valid in practice. Time series of stock market indexes for some major stock exchanges yield empirical examples. In most cases, the statistical approximation to actual dynamic behavior is improved substantially by considering augmented ARCH structures.
\end{abstract}

\section{INTRODUCTION}

For some time now, scientific interest in serially correlated volatility has been soaring. This interest is concentrating primarily on financial time series where prediction of means is notoriously unrewarding and hence structure, if any, is to be found through higher-moments properties only. For many financial price variables, including common stocks 
and stock market indicators, the theory of efficient markets implies that (logarithms of) time series follow random walks. Hence, their first differences are unpredictable while forecasts on the series itself are provided by the latest observation plus a possible 'drift constant'. Empirical evidence typically tends to reject the theory, although the amount of serial correlation in returns is too small and too unstable in time to permit systematic gains by risk-averse investors. A closer look at such series reveals, however, that they show noteworthy temporal clusters of volatility.

The best known statistical model for the volatility-clustering phenomenon is the ARCH model (see Engle, 1982). The acronym denotes 'autoregressive conditional heteroskedasticity', stressing that the model is designed to parallel the central position that the AR, and more general Box-Jenkins, models have in linear time series analysis. The basic model is primarily a model for white noise data without serial correlation structure 'in means'. Problems arising from the reconciliation of linear structure with conditional volatility, already outlined in the original paper by Engle, were tackled in a slightly different way by Weiss (1984). Tsay (1987) and Bera et al. (1992) viewed the problem in the framework of random coefficient models.

Just as the Box-Jenkins ARMA models are built around the archetypal case with Gaussian innovations and this assumption can be substantially relaxed but statistical properties be retained, the typically counterfactual assumption of conditional normality in ARCH models (see Engle, 1982) can be relaxed and ARCH modeling then becomes a powerful descriptive device for parameterizing the fourth-moments structure of time series. This quasi-maximum likelihood or weak ARCH interpretation was previously proposed, explicitly or implicitly, by Weiss (1984), Tsay (1987), and others. In contrast, Engle (1982) and Bollerslev (1986) apparently view the statistical models as approximations to the exact distribution of the data-generating process. However, the latter view implies that one either must restrict attention to the empirically rare Gaussian case or one must develop complicated adaptive estimation techniques. Here, ARCH modeling will be seen as a direct extension of linear ARMA modeling and the first interpretation is adopted.

Even if one restricts attention to cross-moments among squares, such as $\mathrm{E}\left(X_{t}^{2} X_{t-j}^{2}\right)$, modeling conveniently goes along with some linear time series specification as a first step to capture the secondorder moments of type $\mathrm{E}\left(X_{t} X_{t-j}\right)$. ARCH models can then be fitted to the linear innovations. However, it is not clear whether to focus on the moments' structure of the process proper or of its innovations. Engle's (1982) seminal paper appears to have imposed a slight bias 
towards the latter route whereas, for example, Tsay (1987) adopts the former view. In the parametric models used here, modeling is based primarily on innovations moments. However, the influence of higher moments among observed variables and their squares is accounted for, which brings in mixed moments such as $\mathrm{E}\left(X_{t}^{2} X_{t-i} X_{t-j}\right)$.

This paper is organized as follows. Section II reviews the class of augmented ARCH models which encompasses most linear ARCH models known from the literature. Conditions for covariance stationarity are given. Section III presents some simulation results related to strict stationarity of the model class. Section IV is concerned with issues related to the problem of maximum-likelihood estimation of the parameters of the structures. Analytical derivatives are given in the appendix. Section V reports the results from fitting the more general ARCH model class to the Standard \& Poor 500 Index series and to other selected stock index time series. The findings indicate that some real-life ARCH structures are richer than allowed by the restrictive classical ARCH models. Section VI concludes.

\section{THE MODEL CLASS CONSIDERED}

We conceive the data $y_{t}$ as realizations of a stochastic process. We assume we can approximate linear behavior reasonably well by a time series model with innovations $\varepsilon_{t}$. This includes white noise $y_{t}=\varepsilon_{t}$ and the random walk $y_{t}=y_{t-1}+\varepsilon_{t}$ as special cases.

The model class considered for $\varepsilon_{t}$ has the following form

$$
\mathrm{E}\left(\varepsilon_{t}^{2} \mid I_{t-1}\right)=h_{t}=a_{0}+\sum_{i=1}^{R} \sum_{j=1}^{i} a_{i j} \varepsilon_{t-i} \varepsilon_{t-j}
$$

with possibly $R=\infty$. $I_{t}$ denotes an information set containing all $\varepsilon_{s}$ for $s \leq t$. In contrast to linear time series analysis, this $I_{t}$ is nonlinear in the sense that it contains information on $\varepsilon_{s}$ as well as on the cross-products $\varepsilon_{s} \varepsilon_{s-i}$. Expectation is still linear with respect to these cross-products. This model class corresponds closely to the AARCH and GAARCH models of Bera et al. (1992) who derived them from a random coefficient structure, and to the CHARMA models by Tsay (1987) who adopted the converse view to (2.1) and let conditional heteroskedasticity depend exclusively on observed variables. Consequently, the stationarity conditions for CHARMA models (and also for GAARCH) are similar to those given in the following.

In order that Equation (2.1) should make sense, it must obey certain restrictions. In particular, the following assumptions, 1 and 2 , ensure that $h_{t}$ is a well-defined conditional variance process with $h_{t}>0$ 
with probability one. Assumption 3 is needed to establish the existence of a stationary solution to Equation 2.1. It could be replaced by requiring simply that $\Sigma a_{i i}$ converge to a limit smaller than one. Assumptions 4 and 5 describe the stochastic process $\varepsilon_{t}$ and may potentially be replaced by weaker conditions.

Assumption 1. $a_{0}>0$.

Assumption 2. The array $\mathbf{B}$, for convenience, formed from the coefficients $a_{i j}$ in such a way that

$$
\begin{aligned}
b_{i i} & =a_{i i} \\
b_{i j} & =a_{i j} / 2 \text { for } i>j \\
b_{i j} & =b_{j i} \text { for } i<j
\end{aligned}
$$

is non-negative definite in the sense that all finite-dimensional principal submatrices are non-negative definite.

Assumption 3a. The roots of the polynomials in the sequence of characteristic polynomials formed by the diagonal elements of $\mathbf{B}$ are bounded away from the unit circle, i.e. the modulus of any root is greater than $1+\delta$ for some $\delta>0$.

Assumption 3b. The sum $\Sigma a_{i i}=a$ converges.

Assumption 4. $\varepsilon_{t}$ has an MDS (martingale difference series) property in the sense that

$$
\mathrm{E}\left(\varepsilon_{t} \mid I_{t-1}\right)=0 \text { for all } t
$$

Assumption 5. The conditional distribution of $\varepsilon_{t}$ given $I_{t-1}$ is normal.

Even though Assumptions 1-4 would generally suffice to establish the following results together with some more general regularity conditions, I will assume for the moment that Assumption 5 holds in order to facilitate the presentation.

Proposition 1. Under Assumptions 1-5, Equation 2.1 has a covariancestationary solution

A sketch of the proof of Proposition 1 is given in the appendix. Note in particular that stationarity is unaffected by off-diagonal elements in B. Off-diagonals in $\mathbf{B}$ are, however, severely restricted by non-negative definiteness and hence the diagonal of $\mathbf{B}$ typically dominates the array. Note that a covariance-stationary solution to Equation 2.1 does not necessarily imply a covariance-stationary solution for $\varepsilon_{t}^{2}$, as fourth-order moments may not be finite. For ARCH processes, conditions for the existence of higher-order moments were developed, Milhøj (1985). Kunst (1997) provides conditions for finite fourth moments in augmented ARCH processes. Violation of these conditions 
is encountered frequently in empirical applications and may adversely affect the properties of estimators.

The model class (Equation 2.1) comprises most linear heteroscedastic models presented in the literature. The original ARCH model by Engle (1982) has only finitely many $a_{i i}$ different from 0 and $a_{i j}=0$ for $i \neq j$. Definiteness holds if $a_{i i} \geq 0$ as $\mathbf{B}$ is a diagonal matrix. The GARCH model by Bollerslev (1986) has infinitely many nonzero $a_{i i}$ which are, however, finitely parameterized by a ratio of lag polynomials. Again, all off-diagonals are 0 and definiteness is easily checked by the diagonal elements. Positiveness of all non-zero polynomial coefficients is not necessary for Assumption 2 to hold but is usually assumed for simplicity or for numerical reasons. In spite of its widespread popularity in empirical finance, the model has some widely ignored drawbacks. For instance, the GARCH model is unable to capture MA-type second-order structures with $\operatorname{corr}\left(\varepsilon_{t}^{2}, \varepsilon_{t-i}^{2}\right)=0, i>i_{0}$. Product processes as suggested by Taylor (1986) may be the more natural model class to consider.

In relation to serially correlated time series, a more interesting special case of Equation 2.1 is the univariate version of the time series ARCH model suggested by Kunst and Polasek (1994), who assume that $\varepsilon_{t}$ is the innovations process from a linear time series model for the data $y_{t}$ and that $h_{t}$ depends on lagged squared mean-adjusted observations

$$
h_{t}=a_{0}+\sum_{i=1}^{R} a_{i}\left(y_{t-i}-\mu\right)^{2}
$$

If all coefficients are positive, then $\mathbf{B}$ is again n.n.d. In the ARMAARCH model by Weiss (1984), $h_{t}$ depends on both lagged squared observations and lagged squared innovations:

$$
h_{t}=a_{0}+\sum_{i=1}^{R} a_{i}\left(y_{t-i}-\mu\right)^{2}+\sum_{i=1}^{s} c_{i} \varepsilon_{t-i}^{2}
$$

In Equation 2.5, definiteness is ensured by $a_{i} \geq 0, c_{i} \geq 0$. Stationarity conditions in Equations 2.4 and 2.5 depend on the properties of the linear time series process. There is a trade-off in the sense that more linear dependence can be tolerated if the coefficients $a_{i}$ and $c_{i}$ are sufficiently small. Theoretically, some non-negativity conditions could also be relaxed. 


\section{STRICT STATIONARITY IN ARCH GENERALIZATIONS}

Whereas assumptions 1-4 are more or less minimal conditions for covariance stationarity, strict stationarity may hold in more general circumstances. Nelson (1990) has shown that conditional normality allows for strictly stationary solutions of certain models of type (2.1) not only for $a=1$ in Assumption 3b but also for considerably larger values. A similar behavior was found by Kunst (1993) for certain bivariate ARCH structures. In those cases, unconditional variances do not exist. Bougerol and Picard (1992a) provide an extension to higher-order GARCH models.

Whereas conditions for covariance stationarity typically come in the shape of eigenvalue restrictions such as Assumption 3a- e.g. compare Theorem 2 of Engle (1982) or Proposition 1 of Bera et al. (1992)conditions for strict stationarity are rooted in evaluations of the spectral radius and depend on distributional assumptions. Only in very simple cases - see Nelson (1990) - do such evaluations admit closedform conditions. Results for higher-order models, including the condition on top Lyapunov coefficients by Bougerol and Picard (1992a,b), are accessible through numerical simulation only.

Some ARCH processes can be viewed as random coefficient autoregressions (RCA, see Nicholls and Quinn, 1982) and the stability properties of those processes can be applied. For example, consider the simple non-Engle ARCH model of type (2.4)

$$
\begin{aligned}
y_{t} & =\varphi y_{t-1}+\varepsilon_{t} \\
\varepsilon_{t} & \sim N\left(0,1+a y_{t-1}^{2}\right)
\end{aligned}
$$

For $\varphi=0$, this model is Engle-ARCH and the boundary of the strict stationarity region is given by Nelson's Theorem 6 as $a=3.562 \ldots$ This means that the $\mathrm{ARCH}$ process

$$
\varepsilon_{t} \sim N\left(0,1+3.56 y_{t-1}^{2}\right)
$$

is strictly stationary even though its marginal distribution does not possess any useful moments. For arbitrary $\varphi$, the process (3.1) is equivalent to the RCA process ${ }^{1}$

$$
\begin{array}{ccc}
y_{t} & = & \varphi_{t} y_{t-1}+\varepsilon_{t} \\
\varphi_{t} & \text { i.i.d. } & N(\varphi, a) \\
\varepsilon_{t} & \text { i.i.d. } N(0,1) &
\end{array}
$$

\footnotetext{
${ }^{1}$ Generalizing scales by allowing $\varepsilon_{t}$ to have variance $a_{0}$ does not affect the following arguments.
} 
Stability conditions for such RCA processes have been developed by Bougerol and Picard (1992b) based on a theorem by Brandt (1986). The ensuing conditions yield

$$
\mathrm{E}_{\varphi, a} \log \left|\varphi_{t}\right|<0
$$

This boundary is replicated in Fig. 1. It was corroborated by a simulation experiment. Out of 1000 replications of the borderline processes of length 10,000, around 80 replications transgressed the value of $10^{10}$ in absolute value. This value was therefore taken as a criterion for process stability in other situations where theoretical conditions were intractable. ${ }^{2}$

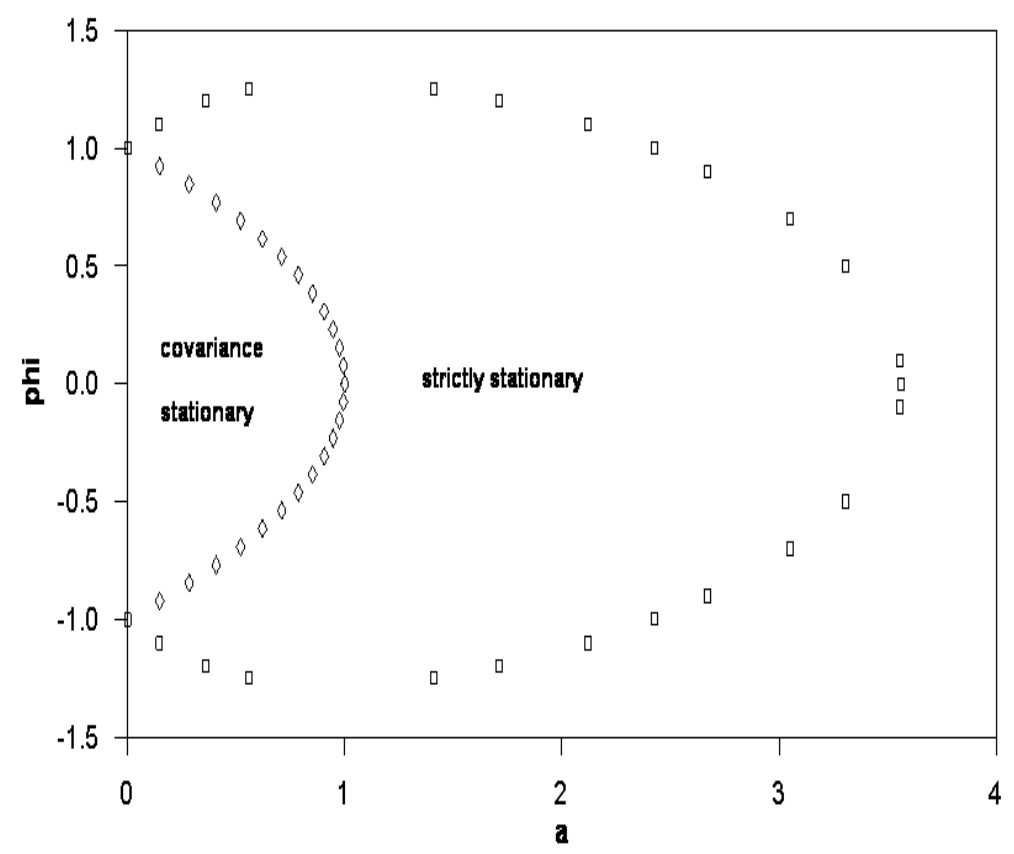

Figure 1: Boundary points of strict and covariance stationarity areas for the model $y_{t}=\varphi y_{t-1}+\varepsilon_{t}, \mathrm{E}\left(\varepsilon_{t}^{2} \mid I_{t-1}\right)=1+a y_{t-1}^{2}$.

From Fig. 1, note that stationarity of Equation 3.1 is critically affected by the ARCH part in such a way that stable processes can evolve from apparently explosive autoregressions. Trajectories of processes in this upper lobe of the stationarity area show a curious behavior, phases of rapid expansion being accompanied by an increase in volatility which eventually leads to an abrupt end of the expansionary phase

\footnotetext{
${ }^{2} \mathrm{~A}$ different and more refined simulation technique was applied by Kleibergen and VanDijk (1993) who replicated Nelson's theoretical boundary for the GARCH(1,1) model.
} 
by hitting upon some smaller number by chance, whereupon the expansion starts anew. However, these processes are of little empirical significance.

The graph is symmetric around the abscissa. For $\varphi \uparrow 1$, the $a$ boundary shifts left only slowly and $a=2$ yields an admissible model for a random walk. Most estimates reported from actual financial returns series point to the central region of the graph where the boundary is almost vertical and the simulated model is close to the EngleARCH model. In the notation of Equation 2.2, the Engle-ARCH model has diagonal $\mathbf{B}$ while the simulated AR-ARCH has Toeplitz $\mathbf{B}$ with geometrically decaying off-diagonals.

Even if - as in some examples reported in Section V-evidence suggests only slow decay in $\mathbf{B}$, the case $\varphi=1$ in Equation 3.1 can be viewed as a limiting case, notwithstanding the true properties of the AR process. Figure 1 shows that the accumulated process $(1-B)^{-1} y$ is stationary if $y$ is white noise with such fourth-order characteristics. Hence, $y$ itself is also stationary for the empirically obtained ARCH parameters. Though this extreme case of non-decaying fourth moments is not generally supported by the data, the implications of such a feature would certainly be interesting. Such a model would imply that the level index (!) under investigation is stationary (or trend-stationary) and that this property is warranted by its extreme reaction to volatility shocks.

The strict stationarity properties of the general model, Equation 2.1 , with $R=2$ were also investigated by simulation experiments. ${ }^{3}$ The following parameterization admits a reasonable graphical representation

$$
h_{t}=1+a_{1} \varepsilon_{t-1}^{2}+a_{2} \varepsilon_{t-2}^{2}+\tau \sqrt{a_{1} a_{2}} \varepsilon_{t-1} \varepsilon_{t-2}
$$

By non-negative definiteness, $\tau$ is restricted to the closed interval $[-1,1] . \tau=0$ yields the Engle-ARCH(2) model. For $\tau=0, \tau=0.5$ , and $\tau=1.0$, simulated boundary curves of the strict stationarity area are presented in Fig. 2. For $\tau<0$, behavior is symmetric to positive $\tau$. Note that, for $\tau=1$, the boundary appears to be nearly

\footnotetext{
${ }^{3}$ In this case, theoretical existence and uniqueness of the stationary distribution obeying the defining equation are not warranted as such. In the covariance-stationary case outlined in section I, these follow from the time-constancy of the generating mechanism and covariance stationarity. In Engle-type ARCH models, they follow from the techniques outlined by Bougerol and Picard (1992a) for positive autoregressions. In Weiss-type models, they follow from the equivalence to random coefficients structures and from the results by Bougerol and Picard (1992b). For the general model, these properties have not been established yet. It is reasonable to assume, however, that ergodic distributions will be well-behaved here and this presumption is also supported by Monte Carlo results.
} 
linear unless $a_{1}$ is very small. Also note that the curves for $\tau=0$ and $\tau=0.5$ are very close to each other, indicating that significant additional ground for the stationarity region is gained for large deviations from the classical model only. $a_{1}=0$ yields the same boundary value for $a_{2}$ as $a_{2}=0$ does for $a_{1}$. Figure 2 shows, however, that behavior with respect to the two parameters is not at all symmetric.

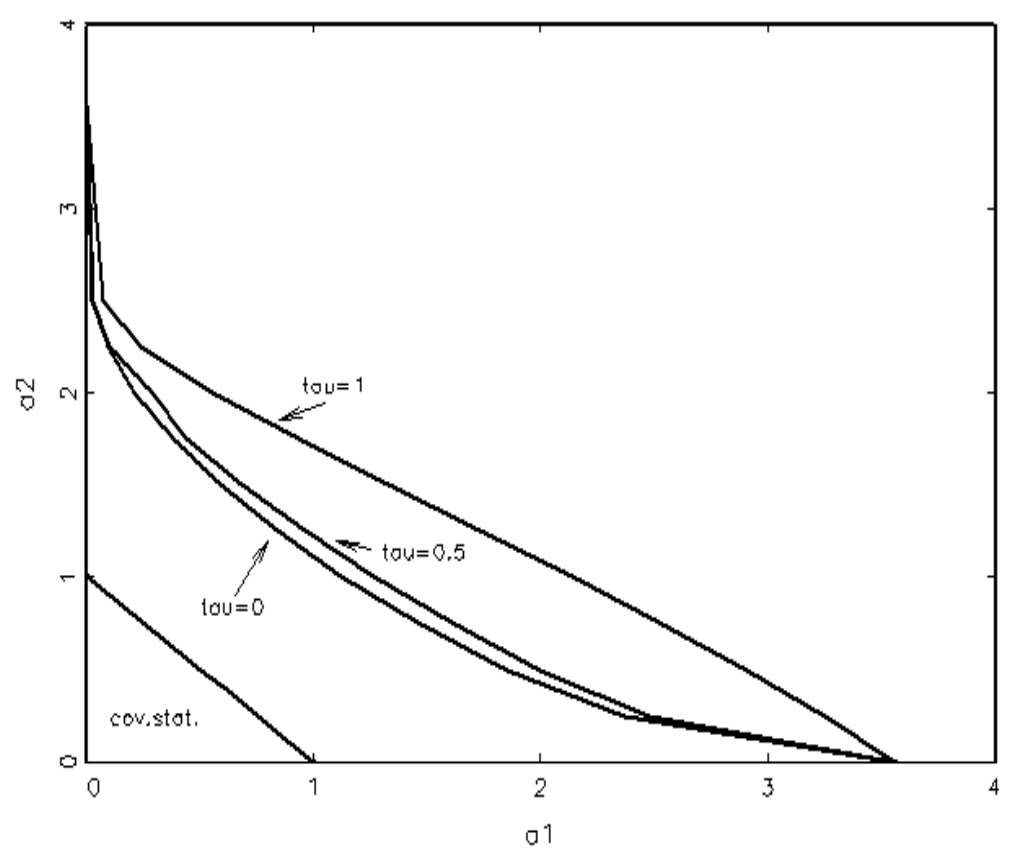

Figure 2: Simulated boundaries of strict and covariance stationarity areas for the model $y_{t}=\varepsilon_{t}, \mathrm{E}\left(\varepsilon_{t}^{2} \mid I_{t-1}\right)=1+a_{1} \varepsilon_{t-1}^{2}+a_{2} \varepsilon_{t-2}^{2}+\tau \sqrt{a_{1} a_{2}} \varepsilon_{t-1} \varepsilon_{t-2}$. Points to the right correspond to non-stationary models.

It evolves from these and some further (unreported) experiments that, firstly, with increasing ARCH lag order, the area between the strictly-stationary and the covariance-stationary boundaries shrinks, and, secondly, this area grows if off-diagonal $a_{i j}$ are present as compared with the classical Engle-ARCH model.

\section{ESTIMATION ISSUES}

Here and in the following it will be assumed that $R<\infty$. This is not the only way to define a finite parameterization for estimation purposes, and models with infinite-dimensional arrays $\mathbf{B}$ which depend on 
a finite parameter set - such as GARCH models - may be worth considering. To ease presentation, however, I assume that a finite matrix gives a reasonable approximation to the possibly infinite-dimensional B.

All ARCH likelihoods can be expressed in the way outlined by Engle (1982, p.990)

$$
\begin{aligned}
\ell & =T^{-1} \sum_{t=1}^{T} \ell_{t} \\
-\ell_{t} & =\frac{1}{2} \log h_{t}+\frac{1}{2} \varepsilon_{t}^{2} / h_{t}
\end{aligned}
$$

but straightforward numerical optimization of Equation 4.1 can be time-consuming. If $\varepsilon_{t}$ has to be estimated from some time-series model for an observed process $y_{t}, h_{t}$ becomes a complicated function of lags of $y_{t}$ and all parameters, i.e. the ARCH parameters of Equation 2.1 as well as the parameters of the linear time-series model. Moreover, all stability and admissibility restrictions are non-linear inequality constraints that make estimation even more cumbersome.

An important simplification can be obtained if the information matrix is block-diagonal in the sense that there is no interaction between parameters of the linear time series model $\theta_{1}$ and $\theta_{2}=\left(a_{0}, a_{11}, a_{21}, a_{22}, \ldots\right)^{\prime}$ of Equation 2.1. In that case, solution of the ML problem can be decomposed into iterative steps of solving for either $\theta_{i}(i=1,2)$ separately, conditional on the most recent parameters of the other $\theta_{i}$ $(i=2,1)$. Engle (1982, Theorem 4) stated some sufficient conditions for this property which he calls symmetry and regularity. Equation 2.1 is Engle-regular in the sense that $h_{t}$ is bounded away from zero by $a_{0}>0$ and that certain expectations of $h_{t}$ derivatives exist. Equation 2.1 is, however, not Engle-symmetric but Bera et al. (1992) showed that block-diagonality of the information matrix evolves from symmetry in a much wider sense. In short, $h_{t}\left(\varepsilon_{t}, \varepsilon_{t-1}, \ldots\right)$ is Bera-symmetric if changing of all $\varepsilon_{s}$ to $-\varepsilon_{s}$ yields the same value while $h_{t}($.$) is Engle-$ symmetric if this property holds for any change of individual $\varepsilon_{s}$ to $-\varepsilon_{s}$.

Computation time can further be shortened by analytically evaluating scores, i.e. derivatives of $\ell_{t}$ with respect to parameters. These can be based on a convenient re-parameterization of Equation 2.1 that allows to replace the complicated admissibility restrictions by nonnegativity constraints. If parameters are expressed through squares, estimation can proceed without further constraints. Formulae for scores and an outline of their derivation are given in the appendix. 


\section{EMPIRICAL EXAMPLES}

\section{Standard \& Poor 500}

A reliable long series frequently used for evaluations of conditionally heteroscedastic models is the Standard and Poor's Index S\&P 500. Daily observations during the time period from 2 July 1962 to 31 December 1990 permit a sample of $7168 .{ }^{4}$ In the linear time series framework, the logarithm of S\&P comes close to a random walk. In a sample of 7168 , however, even an $R^{2}$ of 0.05 indicates statistical rejection of the pure random walk model. In particular, the differences show significant first- and fifth-order autocorrelation, the latter order corresponding to a frequency of five trading days or a week. In contrast, conditional heteroscedasticity within the series is strong, with $\operatorname{GARCH}(1,1)$ models yielding parameter estimates close to the so-called IGARCH boundary where error variances become infinite even if conditional Gaussianity holds. The rather large sample should allow some insight on whether the more general approach suggested in Equation 2.1 is justified relative to the original ARCH model and whether, for example, restrictions as in the Weiss model hold if the standard ARCH appears insufficient.

First, a linear first-order autoregression was tried on the differenced series $y_{t}$ which was amalgamated with a conditionally heteroscedastic structure of type (2.1) with an upper matrix bound of $R=2$. The following parameter estimates were obtained by straightforward optimization of the likelihood

$$
\begin{aligned}
& y_{t}=0.00014+0.185 y_{t-1}+\varepsilon_{t} \\
& h_{t}=0.000019+0.490 \varepsilon_{t-1}^{2}+0.126 \varepsilon_{t-1} \varepsilon_{t-2}+0.426 \varepsilon_{t-2}^{2}
\end{aligned}
$$

According to the estimated Hessian matrix, all six parameters are significant at $1 \%$ and, though the standard $\mathrm{ARCH}$ structure dominates, 0.126 represents a noteworthy off-diagonal element.

The next model estimated was a second-order autoregression with an Equation 2.1 structure with $R=3$ superimposed. The estimated structure was

$$
\begin{aligned}
y_{t}= & 0.00016+0.177 y_{t-1}-0.026 y_{t-2}+\varepsilon_{t} \\
h_{t}= & 0.000019+0.218 \varepsilon_{t-1}^{2}+0.214 \varepsilon_{t-1} \varepsilon_{t-2}+0.362 \varepsilon_{t-2}^{2} \\
& +0.496 \varepsilon_{t-1} \varepsilon_{t-3}-0.056 \varepsilon_{t-2} \varepsilon_{t-3}+0.354 \varepsilon_{t-3}^{2}
\end{aligned}
$$

\footnotetext{
${ }^{4}$ All subsequent analysis is related to these first log differences which are therefore simply called 'the S\&P Index series'.
} 
These coefficients are all significant. The third entry of the diagonal matrix in the re-parameterized form, however, turned out to be insignificant. Hence, the above model contains nine parameters. The restriction $d_{3}=0$ was corroborated by restricted re-estimation.

For $R=4$ and $R=5$, numerical convergence could only be achieved after imposing zero restrictions. $R=5$ is an interesting specification as it accommodates for day-of-the-week effects which are particularly notable from the autocorrelation function of squared returns. After some trial and error, the following model was found to have satisfactory numerical properties. This restricted model explains current volatility by one linear combination of previous errors and some previous squared errors. It can be interpreted as showing five "factors": the linear combination which does not correspond to the linear part of the model; and four distinct Engle-ARCH-type lags

$$
\begin{aligned}
y_{t}= & \mu+\varphi_{1} y_{t-1}+\varphi_{2} y_{t-2}+\varphi_{3} y_{t-3} \\
& +\varphi_{4} y_{t-4}+\varphi_{5} y_{t-5}+\varepsilon_{t} \\
h_{t}= & a_{0}+d_{1}\left(\varepsilon_{t-1}+l_{21} \varepsilon_{t-2}+l_{31} \varepsilon_{t-3}+l_{41} \varepsilon_{t-4}+l_{51} \varepsilon_{t-5}\right)^{2} \\
& +d_{2} \varepsilon_{t-2}^{2}+d_{3} \varepsilon_{t-3}^{2}+d_{4} \varepsilon_{t-4}^{2}+d_{5} \varepsilon_{t-5}^{2}
\end{aligned}
$$

Parameter estimates for Equation 5.3 are given in Table 1 (penultimate column). For unrestricted models, estimation yielded unsatisfactory results. The iteration process became increasingly lengthy and failed to converge or converged to unstable solutions with many insignificant coefficients. This behavior may be related to the aforementioned identification problems.

In summary, neither the restriction suggested by the Engle-ARCH model nor the other extreme Equation 2.4 were supported. Off-diagonal elements were significant and their size was considerable. The decay versus the south-west (or north-east) corner of $\mathbf{B}$ is much slower than would be prescribed by Equation 2.4.

Although I use no explicit Bayesian framework, I adopt the view that fitting models to data is an exercise of optimum approximation within classes of models rather than estimation of true structures. Having abandoned the idea that the S\&P Index really follows a conditionally Gaussian ARCH model, many specification tests do not make sense any more as the concept of 'misspecification' becomes an empty word. However, one may still be interested in the sensitivity of relative advantages of models with respect to changes of the sampling intervals.

Hence, not to 'test' but to check on the stability of Equation 5.3 with respect to time-heterogeneity, Equation 5.3 was re-estimated for subsamples. The selection of subsamples was inspired by Hauser and 
Table 1: Coefficient estimates from general ARCH model (4.3) and from Engle-ARCH model for Standard 83 Poor 500 Index series based on subsamples.

\begin{tabular}{ccccccccc}
\hline & $1962-67$ & & $1969-78$ & & $1979-86$ & & $1962-90$ & \\
\hline$\sqrt{d_{1}}$ & 0.601 & 0.627 & 0.500 & 0.504 & 0.175 & 0.175 & 0.529 & 0.533 \\
$\sqrt{d_{2}}$ & 0.218 & 0.103 & 1.200 & 0.211 & 0.424 & 0.120 & 0.342 & 0.087 \\
$\sqrt{d_{3}}$ & 0.183 & 0.008 & 0.473 & 0.286 & 0.731 & 0.860 & 0.254 & 0.176 \\
$\sqrt{d_{4}}$ & 0.002 & 0.224 & 0.007 & 0.286 & 0.581 & 0.027 & 0.355 & 0.541 \\
$\sqrt{d_{5}}$ & 0.172 & 0.370 & 0.617 & 0.521 & 0.075 & 0.098 & 0.246 & 0.294 \\
$l_{21}$ & -0.220 & & -0.514 & & -0.805 & & -0.568 & \\
$l_{31}$ & 0.383 & & -.263 & & 0.352 & & 0.361 & \\
$l_{41}$ & 0.197 & & 0.447 & & -0.013 & & -0.110 & \\
$l_{51}$ & 0.218 & & 0.746 & & 0.117 & & 0.252 & \\
$\sqrt{a_{0}}$ & 0.00311 & 0.00023 & 0.00000 & 0.00522 & 0.00066 & 0.00002 & 0.00392 & 0.00163 \\
$\mu$ & 0.00023 & 0.00035 & 0.00001 & 0.00010 & 0.00043 & 0.00043 & 0.00021 & 0.00031 \\
$\varphi_{1}$ & 0.197 & 0.170 & 0.295 & 0.302 & 0.094 & 0.094 & 0.191 & 0.194 \\
$\varphi_{2}$ & 0.045 & 0.063 & -.098 & -.103 & 0.002 & 0.002 & -.062 & -.060 \\
$\varphi_{3}$ & -.003 & 0.012 & 0.039 & 0.047 & -.016 & -.016 & -.022 & -.016 \\
$\varphi_{4}$ & -.041 & -.026 & 0.022 & 0.023 & -.020 & -.021 & 0.027 & 0.026 \\
$\varphi_{5}$ & 0.028 & 0.041 & -.037 & -.037 & 0.008 & 0.007 & 0.015 & 0.014 \\
$\sum a_{i i}$ & 0.573 & 0.591 & 2.567 & 0.734 & 1.112 & 0.795 & 0.796 & 0.702 \\
$-2 \ell$ & 13202 & 13183 & 21751 & 21746 & 17102 & 17102 & 61573 & 61559 \\
\hline
\end{tabular}


Kunst (1993) who worked with the same data: a first subsample covers the early years until 1968 which year, however, was excluded because of its irregularities; a second subsample lasts from 1969 through 1978, i.e. the following decade; a third subsample starts in 1979 and ends in 1986, i.e. the year before the 'Black Monday' crash. Table 1 gives estimates for these subsamples and for the whole sample according to model (5.3). Also the results from estimating the same models under the restriction $l_{21}=l_{31}=l_{41}=l_{51}=0$ are provided, i.e. from estimating a pure Engle-type $\mathrm{ARCH}(5)$ model.

Assuming all regularity conditions to hold, the likelihood ratio for testing the restricted Engle-ARCH model against the more general form (Equation 5.3) would be $\chi^{2}$-distributed with four degrees of freedom. The restriction would be rejected for the whole sample and for the early years but not for the other two subsamples. This means that rejection of the Engle-ARCH model is primarily rooted in the early years and in the crash year 1987. Estimation of subsamples around 1987 enhanced this conclusion (these results are probably not very interesting on their own and therefore not reported). For the years 1969-78, unrestricted estimation resulted in an unsatisfactory model which did not meet covariance-stationarity conditions. Except for the general structure identified from the time range 1979-86 which, however, violated covariance-stationarity boundaries only slightly, all other estimated models are stationary.

Many coefficient estimates differ quite a lot among subsamples, but some features were remarkably stable. Firstly, in the lag pattern of ARCH coefficients, $d_{1}$ dominates (except 1979-86) and $d_{4}$ is the least significant in many specifications. Secondly, $l_{21}<0$ in all cases while $\varphi_{1}>0$, implying that the time series factor in Equation 5.3 is not the same as the series itself and hence the data cannot be described by a structure such as Equation 2.4. In summary, the structure is weak in the Standard \& Poor 500 Index - this is not surprising and not very new either-but the little structure that was found can be explained by neither of the two simplified models, i.e. the Engle-ARCH and the (2.4) model, though the Engle-ARCH may be the less detrimental simplification.

In two cases, covariance-stationarity conditions were not met. In Section III, the feature of strict stationarity - as opposed to covariance stationarity - was treated and it was shown that it typically requires less stringent conditions. Hence, the model estimated for 1979-86 is probably still strictly stationary (while the estimated model for 196978 would probably be too far away from the stationarity boundary to meet even more liberal conditions). Simulations of these structures, however, reveal that the behavior implied by these variance-free 
strictly stationary processes is not reflected in the Standard \& Poor 500 Index. Therefore non-stationary structures - in the sense of covariance stationarity - should be seen as implausible models and as indicating a flaw in the specification of Equation 5.3.

\section{A German stock market index}

The German stock market index provided by Morgan Stanley Capital International (MSCI) used here has 2843 observations starting at 1 August 1984, and ending at 13 November 1995. Logarithmic differences of the original series are analyzed. Linear time dependence is weak but autocorrelations are statistically significant up to a lag of seven. A linear autoregressive model of order five appears to capture most of this low-order serial correlation structure. Just as in the Standard \& Poor Index, serial correlation in the squared residuals is more substantial. This second-order correlation is low at lag three but then increases again and is still felt at lag lengths exceeding one trading week.

In order to use the likelihood optimization algorithm, extremely insignificant elements have to be eliminated a priori to avoid numerical difficulties. A good exploratory tool is linear least-squares regression of the squared residuals on lagged squares and cross-products. The thirdorder diagonal element appeared to be very small and the following model structure was used for non-linear optimization:

$$
\begin{aligned}
y_{t} & =\mu+\sum_{i=1}^{5} \varphi_{i} y_{t-i}+\varepsilon_{t} \quad \mathrm{E}\left(\varepsilon_{t}^{2} \mid I_{t-1}\right)=\alpha_{0}+\varepsilon_{-}^{\prime} \mathbf{L D L}^{\prime} \varepsilon_{-} \\
\mathbf{L} & =\left[\begin{array}{ccccc}
1 & 0 & 0 & 0 & 0 \\
l_{21} & 1 & 0 & 0 & 0 \\
l_{31} & l_{32} & 1 & 0 & 0 \\
l_{41} & l_{42} & 0 & 1 & 0 \\
l_{51} & l_{52} & 0 & l_{54} & 1
\end{array}\right] \\
\mathbf{D} & =\operatorname{diag}\left(d_{1}, d_{2}, 0, d_{4}, d_{5}\right)
\end{aligned}
$$

This statistical model contains 19 parameters and the corresponding estimates are given in Table 2. Note that, due to the preliminary search procedure, standard errors are not valid and have been omitted. It should be mentioned, however, that almost all coefficients turn out to be significantly different from zero if the usual guideline is followed, including the $l_{i j}$ for $i \neq j$ that discriminate the augmented ARCH from the standard ARCH model.

This result is confirmed by a comparison with a fifth-order standard ARCH model. The difference in the log likelihood is extremely 
Table 2: Coefficient estimates from general ARCH model (4.3) and from Engle-ARCH model for Stock Price Indexes from several countries.

\begin{tabular}{ccccccccc}
\hline & Germany & & France & United Kingdom & Japan & \\
\hline$\sqrt{d_{1}}$ & 0.279 & 0.263 & 0.346 & 0.318 & 0.239 & 0.243 & 0.428 & 0.438 \\
$\sqrt{d_{2}}$ & 0.520 & 0.211 & 0.061 & 0.085 & 0.591 & 0.475 & 0 & 0.513 \\
$\sqrt{d_{3}}$ & 0.153 & 0.125 & 0.236 & 0.266 & 0 & 0.115 & 0.348 & 0.189 \\
$\sqrt{d_{4}}$ & 0 & 0.614 & 0 & 0.051 & 0.085 & 0.232 & 0.331 & 0.159 \\
$\sqrt{d_{5}}$ & 0.024 & 0.895 & 0.332 & 0.297 & 0 & 0.174 & 0.477 & 0.151 \\
$l_{21}$ & 0.117 & & -0.032 & & 0.225 & & 0.599 & \\
$l_{31}$ & -0.394 & & 0.009 & & 0.030 & & -0.173 & \\
$l_{41}$ & -0.298 & & 0.131 & & -0.142 & & -0.103 & \\
$l_{51}$ & 0.422 & & 0.027 & & -0.022 & & 0.171 & \\
$l_{32}$ & 0.117 & & 0.098 & & -0.041 & & & \\
$l_{42}$ & -0.394 & & 0.196 & & -0.139 & & & \\
$l_{52}$ & -0.298 & & -0.005 & & 0.079 & & -0.042 & \\
$l_{43}$ & & & 0.037 & & & & 0.118 & \\
$l_{53}$ & & & 0.003 & & & & 0.205 & \\
$l_{54}$ & 0.422 & & & & 0.024 & & \\
$\sqrt{a_{0}}$ & 0.00160 & 0.00497 & 0.00218 & 0.00187 & 0.00372 & 0.00992 & 0.01434 & 0.00043 \\
$\mu$ & 0.00023 & 0.00042 & 0.00040 & 0.00034 & 0.00043 & 0.00047 & -0.00001 & 0.00046 \\
$\varphi_{1}$ & 0.044 & 0.059 & 0.246 & 0.223 & 0.081 & 0.084 & 0.198 & 0.187 \\
$\varphi_{2}$ & 0.016 & 0.022 & -0.007 & 0.010 & 0.015 & 0.015 & -0.081 & -0.090 \\
$\varphi_{3}$ & -0.015 & -0.009 & -0.036 & -0.033 & -0.012 & -0.013 & 0.008 & -0.001 \\
$\varphi_{4}$ & 0.032 & 0.029 & 0.051 & 0.014 & -0.006 & -0.007 & 0.035 & 0.037 \\
$\varphi_{5}$ & 0.027 & 0.028 & 0.012 & 0.010 & -0.001 & -0.002 & -0.048 & -0.045 \\
$\sum a_{i i}$ & 0.566 & 1.307 & 0.292 & 0.270 & 0.427 & 0.387 & 0.727 & 0.540 \\
$-2 \ell$ & 44953 & 44891 & 45837 & 45832 & 48505 & 48503 & 44596 & 44314 \\
\hline & & & & & & & &
\end{tabular}


significant if the usual asymptotic chi-square guideline - here with eight degrees of freedom - is adopted. Note, however, that the models are incompletely nested because of the restriction $d_{3}=0$ in the general model. The standard ARCH model is not covariance stationary but it appears to be strictly stationary. It generates peculiar trajectories that do not match the behavior of the observed time series. In contrast, the trajectories generated from the augmented ARCH structure cannot be distinguished visually from the data. Another indicator of the poor approximation by the standard ARCH model is the comparatively large $d_{3}$ estimate that appears to pick up some part of the higher-order structure which is more appropriately reflected in the $l_{i j}$ coefficients of the augmented model.

Although such correspondence between generated data from a fitted model and observed data is likely not sufficient to justify the use of the fitted model, the usage of post-sample simulation techniques is to be appreciated. Many reported statistical models do not produce plausible trajectories and sometimes are unable to produce any trajectories at all. Obviously, these models must be discarded.

It also pays to compare sample moments and other features between artificial and observed data. In the present case, the observed skewness and high kurtosis is not reflected fully in most trajectories of comparable length. Most sample kurtosis values in simulations ranged between 4 and 15, whereas observed kurtosis is 16.4. As in most applications of ARCH models, the conditional distribution in the actual data is non-Gaussian and this causes the slight mismatch.

On the other hand, observed negative skewness cannot be represented in the ARCH framework. If this feature is regarded as relevant, bilinear models may be an obvious suggestion. In contrast, the evidence does not support the ARCH-in-mean model which also generates non-normal third moments from its parametric structure and has a stronger basis in economic theory. This observation also holds for our examples, as was found by some tentative estimation.

\section{A French stock market index}

The MSCI time series on the Paris stock exchange covers the same time range as the German series, from 1 August 1984 to 13 November 1995. Due to different holidays, the French series is slightly longer with 2846 observations. We again analyze the time series of the log differences or approximate returns.

The linear time series structure is weaker than in the German series, and so is the second-order structure in the residuals. Only the first-order lag and some lags exceeding one trading week were found to 
be significant, which may suggest a first-order autoregression instead of the fifth-order autoregression that we have been tentatively using so far. Regarding the ARCH structure, the fourth-order lag turned out to be very small and hence $d_{4}$ was set to zero before conducting maximum likelihood estimation.

Two models are considered in Table 2: first, the most general ARCH model with a fifth-order autoregression, $d_{4}=0$ being the only restriction; secondly, a standard fifth-order ARCH model. It was also attempted to fit a more parsimonious augmented model by eliminating all seemingly insignificant parameters based on a linear first-order autoregression and $d_{2}=d_{4}=0$. However, it turned out that this model involves a substantial deterioration in the quality of fit relative to the first model. On the other hand, the standard ARCH model comes close to the full model. Generated trajectories from all three models are similar to each other and plausibly close to the actual data. In consequence, evidence on the French index does not seem to support the usage of the augmented ARCH model, as the standard ARCH model achieves a reasonable precision.

\section{A British stock market index}

The UK stock market index provided by MSCI covers the time range from 1 August 1984 to 10 November 1995. For the logarithmic differences, there are 2863 observations available.

The British series shows a noteworthy linear autocorrelation around the monthly lag that we are unable to account for, restricting ourselves to low-order models. ARCH effects seem to be somewhat stronger than for France but weaker than in the German or in the Standard \& Poor series. Nevertheless, the data is highly leptokurtic. Hence, a large amount of the leptokurtosis observed cannot be modeled by the parametric ARCH process but rather is rooted in the conditional distribution. In consequence, the efficiency of the maximum likelihood estimation procedure may be adversely affected to a much larger degree than in the other series examined.

Fitting a standard fifth-order ARCH model results in a sum of coefficients of almost 0.4. The first, second, and fourth lag turn out to be the most significant. Curiously, this maximum likelihood result is seriously at odds with the naive least-squares fitting procedure which yielded an insignificant second-order lag. Again, this discrepancy may be due to the high leptokurtosis in the conditional distribution. Anyway, an augmented ARCH model was fitted based on non-zero elements at the places one, two, and four and also on non-zero elements in the corresponding columns of $\mathbf{L}$. A variety of alternative models 
was tried but this design happened to be the optimum with regard to the achieved likelihood.

The results are again given in Table 2 . It is seen that there is no significant difference between the quality of fit of the standard ARCH model fit and of the augmented model. In this regard, the British series resembles the French series. However, due to the high degree of non-normality, the quasi-maximum likelihood procedure appears less reliable.

\section{A Japanese index}

The Japanese series on the MSCI index for the Tokyo stock exchange has 2944 observations from 1 August 1984 to 13 November 1995. We again focus on the logarithmic differences. Also for Japan, the linear correlation structure is weak and the volatility correlation is considerably stronger. However, in this case all dependence is at lags of less than five and evidence on 'misspecification' due to omitted longerrange dependence is much weaker than for the European index series. The second-order ARCH lag appears to be insignificant and was omitted for the specification search within the augmented ARCH model class.

The coefficient estimates corresponding to the best model found are given in Table 2. The general model is significantly better than the restricted classical ARCH model. Note that the Engle-ARCH model contains a noteworthy second-order ARCH lag. It was also tried to add this coefficient to the augmented model but this resulted in no further improvement. Simulations from the identified structures confirmed the inadequacy of the Engle-ARCH model. Trajectories showed linear time trends with only small added perturbations. Simulated augmented ARCH processes had shapes similar to the observed series. Hence, the Japanese index constitutes another example of the potential benefits from considering the more general class.

\section{SUMMARY AND CONCLUSION}

For the important US Standard \& Poor 500 Index and for a Japanese and a German stock index, the evidence has indicated more or less convincingly that fourth-moments structures in financial series may be more complicated than the traditional ARCH model implies. A non-parametric comparison of sample moments (unreported) also supported this result. The statistical evidence presented is stronger than the 'weak evidence on more general structures' found by Tsay (1987) in an exchange rate series. For two other countries, France and the 
United Kingdom, the statistical description achieved by the standard ARCH model appears to be sufficient.

Within the limits of this paper, I restricted attention to crossmoments structures of the form $\mathrm{E}\left(\varepsilon_{t}^{2} \varepsilon_{t-i} \varepsilon_{t-j}\right)$, i.e. to the explanation of volatility by preceding cross-terms. Two points have been neglected intentionally which may deserve further investigation. Firstly, the analysis is strictly limited to Gaussian assumptions. It is well known that innovations in financial time series are typically not conditionally Gaussian but slightly conditionally leptokurtic (compare Baillie and Bollerslev, 1989). Non-normality may affect the efficiency of quasi maximum likelihood estimation and the validity of the chi-square approximation to the likelihood-ratio test.

Secondly, higher-order moments of different form were neglected. In its 'weak ARCH' interpretation, Engle's ARCH model can be seen as the first important attempt to parameterize fourth-order cross structures such as $\mathrm{E}\left(\varepsilon_{t}^{2} \varepsilon_{t-i}^{2}\right)$. The GARCH model by Bollerslev (1986) does the same but uses the rational function approximation in place of the previously used polynomial approximation. The later-developed models by Weiss (1984) and Bera et al. (1992) aim at modeling mixed moments such as $\mathrm{E}\left(\varepsilon_{t}^{2} \varepsilon_{t-i} \varepsilon_{t-j}\right)$. Other generalizations such as "ARCH in mean" are concerned with cross-moments of order three and this track is very much at the center of research at the moment (compare Engle and Lee, 1993). There is an ample field for parameterizations of all kinds of higher-order cross moments and, maybe even more important, empirical findings of these higher-order structures may not be independent of one another. In other words, allowing for non-zero third-order moments may change some of the properties of the models treated in this paper, and these effects have also been neglected. It should be pointed out that the two caveats are interrelated in the sense that additional structure can be searched for by deviating from the assumption of Gaussianity or by sticking to that assumption and parameterizing higher-moments structures. The two paths are probably alternatives and the second one has recently proved to be more fruitful.

The framework presented here necessarily excludes all complicated non-linear models whose popularity is still increasing. We hold the view that, unless convincing theoretical motivations for special nonlinear classes can be found, the application of arbitrary functional forms is not natural from a time-series analytical point of view. If dependence in volatility is investigated, then a full understanding of simple stationary models describing cross moments of order up to four is sure an important point to begin with. 


\section{ACKNOWLEDGEMENTS}

The author wishes to thank Michael Hauser for extensive help with all computer work, the participants of the NBER Time Series Seminar 1993, in particular Ruey Tsay, for helpful comments, and a referee for drawing attention to the comparative empirical application presented here. The usual proviso applies.

\section{References}

[1] Bera, A.K., Higgins, M.L., and Lee, S. (1992) Interaction Between Autocorrelation and Conditional Heteroskedasticity A Random Coefficient Approach, Journal of Business and Economic Statistics 10, 133-142.

[2] Baillie, R.T. and Bollerslev, T. (1989) The Message in Daily Exchange Rates. A Conditional-Variance Tale, Journal of Business and Economic Statistics 7, 297-305.

[3] Bollerslev, T. (1961) Generalized autoregressive conditional heteroskedasticity, Journal of Econometrics 31, 307-327.

[4] Bougerol, P. and Picard, N. (1992a) Stationarity of GARCH processes and of some nonnegative time series, Journal of Econometrics 52, 115-127.

[5] Bougerol, P. and Picard, N. (1992b) Strict Stationarity of Generalized Autoregressive Processes, The Annals of Probability 20, 1714-1730.

[6] Brandt, A. (1986) The stochastic equation $Y_{n+1}=A_{n} Y_{n}+B_{n}$ with stationary coefficients, Advances in Applied Probability 18, 211-220.

[7] Engle, R.F. (1982) Autoregressive conditional heteroskedasticity with estimates of the variance of United Kingdom inflation, Econometrica 50, 987-1007.

[8] Engle, R.F. and Lee, G.G.J. (1993) A Permanent and Transitory Component Model of Stock Return Volatility, Paper presented at the ESEM Meeting, Uppsala.

[9] Hauser, M.A. and Kunst, R.M. (1993) Fractionally Integrated Models With ARCH Errors, Research Memorandum No. 322, Institute for Advanced Studies, Vienna, Austria.

[10] Kleibergen, F. and Van Dijk, H.K. (1993) Non-Stationarity in GARCH Models: A Bayesian Analysis, Journal of Applied Econometrics 8, S41-S61. 
[11] Kunst, R.M. (1993) Stability Conditions for a Bivariate ARCH System Which Is Cointegrated in Mean, Communications in Statistics, Theory and Methods 22, 2941-2953.

[12] Kunst, R.M. (1997) Fourth-order Moments of Augmented ARCH Processes. Communications in Statistics, Theory and Methods, 26, 1425-41.

[13] Kunst, R.M. and Polasek, W. (1994) Structuring Volatile Swiss Interest Rates: Some Evidence on the Present Value Model and a VAR-VARCH Approach, in Econometric Analysis of Financial Markets, Eds J. Kaehler and P. Kugler, Physica-Verlag, Heidelberg.

[14] Lamoureux, C. and Lastrapes, W. (1990) Persistence in Variance, Structural Change, and the GARCH Model, Journal of Business E Economic Statistics 8, 225-234.

[15] Milhøj, A. (1985) The Moment Structure of ARCH Processes, Scandinavian Journal of Statistics 12, 281-292.

[16] Nelson, D.B. (1990) Stationarity and Persistence in the GARCH(1,1) Model, Econometric Theory 6, 318-334.

[17] Nicholls, D.F. and Quinn, B.G. (1982) Random coefficient autoregressive models: an introduction. Lecture Notes in Statistics, Vol. 11, Springer, New York.

[18] Taylor, S. (1986) Modelling Financial Time Series, Wiley.

[19] Tsay, R.S. (1987) Conditional Heteroscedastic Time Series Models, Journal of the American Statistical Association 82, 590-604.

[20] Weiss, A.A. (1984) ARMA models with ARCH errors, Journal of Time Series Analysis 5, 129-143.

\section{Appendix A}

\section{Proof of Proposition 1}

This is perhaps most easily shown if Engle's (1982,p.1005) idea is adopted. First we assume that there is an upper bound to the indices in Equation 2.1, say $R$. Then we stack the elements of concern into an $R(R+1) / 2-$ vector in the following way

$$
\begin{aligned}
w_{t}^{\prime}= & \left(\varepsilon_{t}^{2}, \varepsilon_{t-1}^{2}, \ldots, \varepsilon_{t-R+1}^{2}, \varepsilon_{t} \varepsilon_{t-1}, \varepsilon_{t-1} \varepsilon_{t-2}, \ldots,\right. \\
& \left.\varepsilon_{t-R+2} \varepsilon_{t-R+1}, \varepsilon_{t} \varepsilon_{t-2}, \varepsilon_{t-1} \varepsilon_{t-3}, \ldots, \varepsilon_{t} \varepsilon_{t-R+1}\right)
\end{aligned}
$$

(Alternatively, we could use $\operatorname{vech}\left(\left(\varepsilon_{t}, \ldots, \varepsilon_{t-R+1}\right)\left(\varepsilon_{t}, \ldots, \varepsilon_{t-R+1}\right)^{\prime}\right)$ as a 'state vector' but the present derivation appears more convenient.) 
The vector containing the main diagonal is followed by the elements of the first subdiagonal, then the second subdiagonal etc. Then we can re-write Equation 2.1 as

$$
\mathrm{E}\left(w_{t} \mid I_{t-1}\right)=a_{V}+\mathbf{M} w_{t-1}
$$

with $a_{V}^{\prime}=\left(a_{0}, 0, \ldots, 0\right)$ and with $\mathbf{M}$ constructed as follows

$\begin{array}{cccccccccccccc}a_{11} & a_{22} & \ldots & a_{R-1, R-1} & a_{R R} & a_{21} & a_{32} & \ldots & a_{R-1, R-2} & a_{R, R-1} & a_{31} & \ldots & a_{R, 2} & a_{R, 1} \\ 1 & 0 & 0 & 0 & 0 & 0 & 0 & 0 & 0 & 0 & 0 & 0 & 0 & 0 \\ 0 & 1 & 0 & 0 & 0 & 0 & 0 & 0 & 0 & 0 & 0 & 0 & 0 & 0 \\ 0 & 0 & \ldots & 0 & 0 & 0 & 0 & 0 & 0 & 0 & 0 & 0 & 0 & 0 \\ 0 & 0 & 0 & 1 & 0 & 0 & 0 & 0 & 0 & 0 & 0 & 0 & 0 & 0 \\ 0 & 0 & 0 & 0 & 0 & 0 & 0 & 0 & 0 & 0 & 0 & 0 & 0 & 0 \\ 0 & 0 & 0 & 0 & 1 & 0 & 0 & 0 & 0 & 0 & 0 & 0 & 0 & 0 \\ 0 & 0 & 0 & 0 & 0 & 1 & 0 & 0 & 0 & 0 & 0 & 0 & 0 & 0 \\ 0 & 0 & 0 & 0 & 0 & 0 & \ldots & 0 & 0 & 0 & 0 & 0 & 0 & 0 \\ 0 & 0 & 0 & 0 & 0 & 0 & 0 & 1 & 0 & 0 & 0 & 0 & 0 & 0 \\ 0 & 0 & 0 & 0 & 0 & 0 & 0 & 0 & 0 & 0 & 0 & 0 & 0 & 0 \\ 0 & 0 & 0 & 0 & 0 & 0 & 0 & 0 & 0 & 1 & 0 & 0 & 0 & 0 \\ 0 & 0 & 0 & 0 & 0 & 0 & 0 & 0 & 0 & 0 & \ldots & 0 & 0 & 0 \\ 0 & 0 & 0 & 0 & 0 & 0 & 0 & 0 & 0 & 0 & 0 & 0 & 0 & 0\end{array}$

Apart from the first row, $\mathbf{M}$ contains a sub-diagonal $(R-1) \times(R-1)$ identity matrix filled to the right margin with zeros, then a row of zeros, then another $(R-2) \times(R-2)$ identity matrix, then a row of zeros etc. until a row with a single 1 and then a zero row finish the diagram. The remainder is filled with zeros. Clearly, the asymptotic solution

$$
\lim _{k \rightarrow \infty} \mathrm{E}\left(w_{t} \mid I_{t-k}\right)=(\mathbf{I}-\mathbf{M})^{-1} a_{V}
$$

remains valid. Moreover, this solution represents a covariance-stationary solution if and only if the roots of the characteristic polynomial formed just by the $a_{i i}$ lie outside the unit circle. It is easily seen from the above matrix by expanding along the first row that the remaining $a_{i j}, i>j$ do not affect this property, i.e. the eigenvalues of $\mathbf{M}$ are entirely determined by the $a_{i i}$ elements. Assumption 4 is crucial as otherwise the rows $R+1,2 R, \ldots$ would not necessarily be zero.

Now suppose $R \rightarrow \infty$. Then we can approximate the behavior of $w_{t}$ by that of finitely-structured $w_{R t}$ arbitrarily well and, using Assumption 3, stationarity is established in analogy to the linear autoregressive model.

\section{APPENDIX B}

Scores for the augmented ARCH model 
It is known from linear algebra that any symmetric non-negative definite $R \times R$-matrix $\mathbf{B}$ can be decomposed into $\mathbf{B}=\mathbf{L D L}^{\prime}$ where $\mathbf{L}$ is a lower triangular matrix with a unit diagonal and $\mathbf{D}$ is a diagonal matrix with positive elements and maybe some zeros on that diagonal (Banachiewicz decomposition). Similarly, any $\mathbf{L} \mathbf{D L}^{\prime}$ obeying to these restrictions defines a n.n.d. matrix $\mathbf{B}$ and thus instead of being concerned with the $R(R+1) / 2$ parameters on and below the diagonal in B - which are equivalent to $a_{11}, a_{21}, a_{22}, \ldots, a_{R R}$ in Equation 2.1-we can look at the sub-diagonal elements of $\mathbf{L}$, say $l_{21}, l_{31}, l_{32}, \ldots, l_{R, R-1}$, and at the $R$ elements on the $\mathbf{D}$ diagonal. The n.n.d. constraint has been transformed into a simple non-negativity constraint on the $\mathbf{D}$ elements $d_{1}, \ldots, d_{R}$ and the whole model can be re-written as

$$
h_{t}=\varepsilon_{t-1}^{\prime} \mathbf{L D L}^{\prime} \varepsilon_{t-1}
$$

with $\varepsilon_{t}=\left(\varepsilon_{t}, \ldots, \varepsilon_{t-R+1}\right)^{\prime}$. A disadvantage of the re-parameterization in Equation A.1 is that some elements of $\mathbf{L}$ become unidentified if D has zero elements. In practice, it is suggested to check on the $d_{i}$ via some preliminary Engle-ARCH estimation, i.e., imposing $\mathbf{L}=\mathbf{I}$. If, say, $d_{i}$ is close to $0, d_{i}=0$ and $l_{j i}=0(j=i, \ldots, R)$ can be imposed during the following model selection stages. After finishing these, the significance of $d_{i}=0$ can be re-checked. The empirical results presented below were based on this selection procedure. The advantages sure outweigh this inconvenience.

Further assuming $\varepsilon_{t}$ to be a linear autoregressive transformation of the observed variable vector $\mathbf{y}_{t}$ of length $R+S$, say, $h_{t}$ can also be written in observed variables

$$
h_{t}=\mathbf{y}_{t-1}^{\prime} \mathbf{C}^{\prime} \mathbf{L} \mathbf{D L} \mathbf{C}^{\prime} \mathbf{y}_{t-1}
$$

with $\mathbf{C}$ being an $R \times(R+S)$-matrix with its $i$ th row containing the autoregressive coefficients $\left(1, \zeta_{1}, \ldots, \zeta_{S-1}\right)$ flanked by $i-1$ zeros to the left and filled up with $R-i+1$ zeros to the right. Hence, the derivative with respect to $\zeta_{i}(i=1, \ldots, S-1)$ is

$$
\frac{\partial h_{t}}{\partial \zeta_{i}}=2 \mathbf{y}_{t-1}^{\prime} \mathbf{C}^{\prime} \mathbf{L} \mathbf{D L} \mathbf{y}_{t-i-1}
$$

For technical reasons, the right-hand side $R$-vector $\mathbf{y}_{t-i-1}$ has been trimmed from the end while the left-hand vector $y_{t-1}$ contains $R+S$ elements. Note that for $\mathbf{L}=\mathbf{I}$ the original ARCH case (Engle, p.995) is recovered immediately.

Equation A.3 allows to evaluate the scores with respect to $\zeta_{i}$. In detail,

$$
\frac{\partial l_{t}}{\partial \zeta_{i}}=\frac{\varepsilon_{t} y_{t-i}}{h_{t}}+\frac{1}{2 h_{t}} \frac{\partial h_{t}}{\partial \zeta_{i}} \frac{\varepsilon_{t}^{2}-h_{t}}{h_{t}}
$$


and substitution of Equation A.3 into Equation A.4 yields an operable expression.

Derivatives with respect to the ARCH parameters are more straightforward as $\varepsilon_{t}$ does not depend on them. In detail,

$$
\frac{\partial h_{t}}{\partial d_{i}}=\left(\sum_{j=1}^{R} l_{j i} \varepsilon_{t-j}\right)^{2} \quad \frac{\partial l_{t}}{\partial d_{i}}=\frac{\partial h_{t}}{\partial d_{i}} \frac{1}{2 h_{t}} \frac{\varepsilon_{t}^{2}-h_{t}}{h_{t}}
$$

and similarly

$$
\frac{\partial h_{t}}{\partial l_{i j}}=2 d_{j}\left(\sum_{k=j}^{R} l_{k j} \varepsilon_{t-k}\right) \varepsilon_{t-j} \quad \frac{\partial l_{t}}{\partial l_{i j}}=\frac{\partial h_{t}}{\partial l_{i j}} \frac{1}{2 h_{t}} \frac{\varepsilon_{t}^{2}-h_{t}}{h_{t}}
$$

